Editorial

\title{
Acknowledgment of Reviewers
}

\section{Peter Howe}

Editor-in-Chief of Nutrients, Nutritional Physiology Research Centre, Sansom Institute for Health Research, School of Health Sciences, University of South Australia, Adelaide, South Australia 5001, Australia; E-Mail: peter.howe@unisa.edu.au; Tel.: +61-8-8302-1200; Fax: +61-8-8302-2178

Received: 21 February 2012 / Accepted: 22 February 2012 / Published: 23 February 2012

It is with great appreciation that we acknowledge the following reviewers who have served our research community by reviewing manuscripts for Nutrients in 2011.

$\begin{array}{lll}\text { Stuart L. Abramson } & \text { Anette E. Buyken } & \text { Zora Djuric } \\ \text { Bharat Aggarwal } & \text { Philip Calder } & \text { Fernanda Domingues } \\ \text { Samuel E. Aggrey } & \text { Carlos Camargo } & \text { Marjorie Ellin Doyle } \\ \text { Said Ajlouni } & \text { John J. Cannell } & \text { Gregg Duester } \\ \text { Juan Pablo Albar } & \text { Francesco Cantatore } & \text { Erik Eckhardt } \\ \text { Michael Amling } & \text { Christelle Cebo } & \text { Garry Egger } \\ \text { Amy Anderson } & \text { Hyung-Kyoon Choi } & \text { Susumu Eguchi } \\ \text { John J. B. Andersson } & \text { Sara Cicerale } & \text { Hani S. El-Nezami } \\ \text { Pinarosa Avato } & \text { Milan Ciz } & \text { Lisa Elviri } \\ \text { Giorgio Barbarini } & \text { Karine Clément } & \text { Peter Erickson } \\ \text { Alan Barclay } & \text { Robert Cooney } & \text { François Fenaille } \\ \text { Susan I. Barr } & \text { Anthony Corfield } & \text { Stefano Fiorucci } \\ \text { Sabine Baumgartner } & \text { Robert Cousins } & \text { Julie Fisher } \\ \text { Craig R. Baumrucker } & \text { Georgina Crichton } & \text { Lynda A. Frassetto } \\ \text { Christopher Bell } & \text { Francesca L. Crowe } & \text { William T. Gibson } \\ \text { Ina Bergheim } & \text { Adrian G. Cummins } & \text { Sandra Godden } \\ \text { Marla J. Berry } & \text { Susanna Cunningham-Rundles } & \text { Lourdes Gómez-gómez } \\ \text { Elizabeth R. Bertone-Johnson } & \text { Geraldine Cuskelly } & \text { Michael D. Griswold } \\ \text { Christophe Blecker } & \text { Teresa Lopes Da Silva } & \text { Conny Gysemans } \\ \text { Brian C. Bowker } & \text { Ralph W. De Vere White } & \text { Susan J. Hagen } \\ \text { George Bray } & \text { Eric A. Decker } & \text { Charles Halsted } \\ \text { Thomas Brenna } & \text { Hector Deluca } & \text { Akimasa Hatanaka } \\ \text { Lindsay Brown } & \text { Ulrich Desselberger } & \text { Robert P. Heaney }\end{array}$


David Heber

Jaimie Hemsworth

Paul Hewlett

Allan G. Hill

Chi-Tang Ho

Jonathan M. Hodgson

Michael Holick

Licia Iacoviello

Elena Ibañez

Masahiko Ikeda

Sin-hyeog Im

Filip Van Immerseel

Philippa Jackson

David M. Janicke

Richard J. Johnson

Kieran Jordan

Nathalie Juge

Taiho Kambe

Leila J. Karhunen

Takeshi Katayama

Joseph Katz

Shannon L. Kelleher

Jae Cheol Kim

Todd R. Klaenhammer

Kimberly Kline

Koichi S. Kobayashi

Joanne Kotsopoulos

Laurie L. Lachance

Carl J. Lavie

Derek LeRoith

Selma C. Liberato

Ausgusto Litonjua

Kerry M. Loomes

Alfredo J. Lucendo

Faidon Magkos

Philippe Marteau

Peter Mccaffery

Craig McClain

Gordon J. Mcdougall

Aidan Mcelduff
Lynne V. Mcfarland

Samia Mezouari

Carla K. Miller

Michelle Mitchell

Antonio Morandi

Lee E. Morrow

Mark Moss

Jorge Mujico

Kazutoshi Nakamura

Masanobu Nanno

David C. Nieman

Cristina Novembrino

Rima Obeid

William T. Oliver

Daniel J. O'sullivan

Casey M. Owens

Jeetesh Patel

Simon H. S. Pearce

Francisco J. Pérez-Cano

Vincenzo Pezzi

Eduardo Pimenta

Lindsay D. Plank

Anna Poli

Loredana Quadro

William D. Rees

Manja Reimann

Graziano Riccioni

Amy Riek

Ger T. Rijkers

Joseph F. Robare

Cécile Rochette-Egly

David S. Rosenthal

Ryuji Sakakibara

Francisco J. Sánchez-Muniz

Robert B. Saper

Peter Schemmer

Lutz Schomburg

Michael Schubert

Ulrich Schweizer

Je Kyung Seong
Valeria Sibilia

Gilbert H. Smith

Mario Soares

Danfeng Song

Eliana B. Souto

Ivan Spasojević

Gregory T. Spear

Bonnie Specker

Lyn M. Steffen

Francene M. Steinberg

Kerst Stelwagen

Poul Suadicani

Russell Swerdlow

Michael Symonds

Shahrad Taheri

Francis A. K. Tayie

Carla Taylor

Masaaki Terashima

Reiko Teshima

David Thomas

Diana Thomas

Kieran M. Tuohy

Cuno S. P. M. Uiterwaal

Zeynep Ustunol

Patrícia Valentao

Luca Vangelista

Sandra G. Velleman

Pietro Vernazza

Peter Vestergaard

Michael H. Walter

Connie Weaver

Eric C. Westman

Susan J. Whiting

Martin G. Wilkinson

David S. Wishart

Matthias Wjst

Richard Wood

Kun Zhu

Karin Zitterl-Eglseer 
I also wish to draw your attention on the Nutrients website to our Editorial Board and also to the guest editors of our expanding list of special issues who have devoted their time and expertise to achieving rapid growth in the quantity and quality of published manuscripts. My special thanks also to Associate Editor, Jon Buckley, and members of the editorial office for their dedication to the journal.

(C) 2012 by the authors; licensee MDPI, Basel, Switzerland. This article is an open access article distributed under the terms and conditions of the Creative Commons Attribution license (http://creativecommons.org/licenses/by/3.0/). 